\title{
Perspectiva sobre el pensamiento sistémico
}

\author{
Perspective on systemic thinking
}

DOI: https://doi.org/10.17981/econcuc.41.2.2020.Ensy.2

Ensayo Argumentativo.

Fecha de recepción: 08/06/2020

Fecha de devolución: 27/07/2020

Fecha de aceptación: 12/08/2020

Fecha de publicación: 18/08/2020

\section{Alexandra Camila Vásquez Sarmiento Universidad de la Costa. Barranquilla (Colombia) avasquez19@cuc.edu.co}

Para citar este artículo:

Vásquez, A. (2020). Perspectiva sobre el pensamiento sistémico. Económicas CUC, 41(2), 261-266. DOI: https://doi.org/10.17981/econcuc.41.2.2020.Ensy.2

JEL: B13, D23.

\section{Resumen}

Peter Senge en su libro titulado "La quinta disciplina. El arte y la práctica de la organización abierta al aprendizaje", publicado en 1994, expresa que el pensamiento sistémico es una sensibilidad hacia los entrelazamientos sutiles que confieren a los sistemas vivos su carácter singular, es decir, que el pensamiento sistémico surge de la necesidad de solventar problemas de toda índole, pero, ¿cuáles son los rasgos más importantes del pensamiento sistémico dentro de las organizaciones? El presente ensayo pretende dar respuesta a esta incógnita. La revisión documental sustentó mediante un estado del arte el método, permitiendo concluir que el pensamiento y comportamiento sistémico en conjunto con la conducta humana son claves para lograr que la planeación estratégica sea alcanzada con éxito. Palabras clave: Pensamiento; pensamiento sistémico; conducta humana; cultura organizacional; planeación estratégica

\section{Abstract}

Peter Senge in his book entitled "The fifth discipline. The art and practice of the organization open to learning", published in 1994 expresses that systemic thinking is a sensitivity towards the subtle entanglements that give living systems their singular character, that is, systems thinking arises from the need to solve problems of all kinds, but what are the most important features of systemic thinking within organizations? This essay attempts to answer this question. The documentary review supported the method through a state of the art, allowing to conclude that systemic thinking and behavior in conjunction with human behavior are key to achieve strategic planning successfully. Keywords: Thought, systemic thinking; human conduct; organizational culture; strategic planning 


\section{INTRODUCCIÓN}

Partiendo del concepto de sistema se puede decir que es un conjunto de piezas ordenadas que están organizadas para producir consecuencias; el pensamiento sistémico entonces, trata de entender su actividad para solucionar los inconvenientes que puedan presentar estas piezas. A través del pensamiento sistémico se estudian las partes de un todo, se aplica en estudios científicos, de ingeniería y administración de empresas para dar solución a problemas y situaciones. El Pensamiento sistémico es una sensibilidad hacia los entrelazamientos sutiles que confieren a los sistemas vivos su carácter singular (Senge, 1994).

Surge como la resolución de problemas de organizaciones y de relaciones internacionales, incluso familiares y personales. Se le atribuye su origen al biólogo Ludwig Von Bertalanffy, después se le sumaron otros investigadores de distintas áreas como filosofía, ingeniería, y psiquiatría, los cuales fueron dándole profundidad y desarrollando sus aplicaciones y consecuencias, lo anterior ha sido reseñado por autores de diferentes disciplinas como De la Torre (2018), Paredes-Chacín (2017), Reales (2016) y Henríquez, Rada y Torrenegra (2016).

El filósofo Edgar Morín fue quien se dio a la tarea de pasar del pensamiento sistémico al complejo ya que se caracteriza por ser organizado y desarrollar habilidades que pueden atender diversos problemas filosóficos, científicos y sociales. El Pensamiento Sistémico se ha desarrollado a partir de mediados del siglo XX, sus precursores son los fundadores del Mental Research Institute de Palo Alto en California, EEUU, de allí se expandió a todo el mundo, como disciplina adecuada a la resolución de problemas personales, familiares, de organizaciones pequeñas y grandes, y hasta de relaciones internacionales (Morín, 2004).

\section{Características y metodología del Pensamiento Sistémico}

El Pensamiento Sistémico se caracteriza por ir más allá de las cosas que se perciben a simple vista, de aquí nace su complejidad, por eso puedo nombrar algunos puntos que lo definen:

1. Parte de lo particular a lo general

2. Tiene un punto de vista más complejo

3. Da a conocer patrones, estrategias para sortear situaciones venideras

4. Útil para liderar proyectos

5. Sus estrategias y métodos tienen mayor eficacia en la vida práctica.

De acuerdo a Jaimes et al. (2009) la metodología que emplea se caracteriza por cuatro pasos:

1. Visión global en el cual se pude observar todo de manera general.

2. El Balance del corto y largo plazo en el cual se lleva a través del camino del éxito y va a depender de las implicaciones que se presente. 
3. El reconocimiento de los sistemas dinámicos complejos e interdependientes en el cual a través de herramientas se construye modelos para las situaciones bajo observación.

4. El reconocimiento de los elementos medibles y no medibles el cual el pensamiento sistemático impulsa el correcto uso de indicadores cualitativos y cuantitativos a través de un análisis de la situación.

\section{Naturaleza del pensamiento sistémico}

Al planteamiento ¿Qué podemos esperar del pensamiento sistémico? No hay respuestas correctas, como la dinámica de sistemas ilustra las interdependencias del sistema actual. Nunca existe una sola respuesta correcta para una pregunta. En cambio, la disciplina releva que existe una gran variedad de actos posibles, algunos de los cuales conducen a cambios profundos otros actos producen, casi inevitablemente. Algunas consecuencias no deseadas en otro sector del sistema. El arte del pensamiento sistémico consiste, entre otras cosas, en evaluar las consecuencias del acto que escogemos (Ackoff, 2002).

\section{El pensamiento sistémico en la administración de empresas}

Partiendo de la idea de que una empresa es un sistema de partes interdependientes que interactúan entre sí para lograr un objetivo, el pensamiento sistémico permite visualizar la complejidad de un sistema, comprender su estructura y cómo está compuesta, permitiendo identificar las áreas donde la interacción está siendo interrumpida afectando la productividad de la organización.

Tener una visión global del sistema de una organización nos permite ver más claramente el impacto que estas interacciones están llevando a través de los resultados y como mejorarlos, incorporando nuevas herramientas logrando una dinámica más eficiente (Paz, Harris y Franco, 2016; Rojas, 2016; Chang, 2016a; Chang, 2016b). Mirar la organización en sus diferentes funciones, contextos, interacciones, así con sus actividades internas, externas, sociales e incluso de responsabilidad comunicacional frente al Estado (Hernández, Alvarado y Velazco, 2013; Chumaceiro y Hernández, 2012; Hernández y Buitrago, 2017; Hernández, Meléndez, Chumaceiro y Gil, 2017; Ramírez, Rincón, Chacón y Alemán, 2018).

El autor Russell Linconl Ackoff (2007), quien fue uno de los pioneros en el campo de la investigación de operaciones, el pensamiento sistémico y la ciencia de la administración, realizó grandes atribuciones a la planificación estratégica, partiendo de unos principios, los cuales son:

- Principio participativo.

- Principio de comunidad.

- Principios holísticos. 
A su vez, definió unas fases para lo que el denominó modelo de planeación interactivo, que definiremos a continuación:

- Formulación de la problemática: Planteamiento de amenazas y oportunidades a lo que la organización se vería expuesto.

- Análisis del sistema (Una descripción del estado actual de la organización y su influencia con el medio ambiente).

- Análisis de las obstrucciones (Identifica el modo en cómo se gestionan los conflictos).

- Preparación de proyecto de referencia: Es la comparación entre el pasado el futuro de la organización. En esta fase se revisan las exceptivas de la organización, su conducta actual y su proyección hacia el futuro para determinar que acciones de hoy pueden afectar más adelante la organización.

- Planeación de los fines: Se proyecta un futuro más real y medible en el que se emplean unos pasos:

- Selección de la Misión.

- Especificación de las propiedades deseadas del diseño.

- Diseño del sistema.

- Planeación de los medios: De que manera vamos a llegar o aproximarnos a ese futuro planteado.

- Planeación de los recursos: En esta fase partimos por hacernos la siguiente pregunta: ¿Qué necesito para cumplir estos objetivos y como lo haré?

- Diseño de la implantación y el control: En esta fase requerimos delegar quien va controlar las operaciones en cada área, ya que es muy importante que cada parte del sistema esté operando según lo planeado para evitar consecuencias, así mismo recibir retroalimentación.

- El sistema y su medio ambiente: En esta fase Ackoff plantea que nuestro futuro depende más de las acciones que tomamos hoy, que de lo que hicimos en el pasado.

\section{Conclusiones}

La orientación e interés por el enfoque sistémico y organizacional le permitieron a Ackoff realizar importantes aportes a la planeación estratégica de las organizaciones, analizó el comportamiento sistemático y lo relacionó con la conducta humana permitiendo crear sistemas con propósitos, impulsando la planeación idealizada, formas de administración, considerando siempre aspectos sociales, culturales y psicológicos para lograr el éxito en el desarrollo de un sistema como organización. 


\section{Reconocimientos y Agradecimientos}

Este ensayo es producto de proyecto de aula del área de conocimiento: Talento Humano y Estrategia Empresarial, de la asignatura: Direccionamiento Estratégico, del Programa de Administración de Empresas, del Grupo de Investigación: Administración Social de la Universidad de la Costa.

\section{REFERENCIAS}

Ackoff, R. (2007). Conceptos básicos de la gerencia sistémica. Gotas de Conocimiento, $1-12$.

Ackoff, R. (2002). El paradigma de Ackoff. Una administración sistémica ( $1^{a}$ ed). México, D. F.: Limusa.

Chang, E. (2016a). Pensamiento estratégico en la gestión de las tiendas de barrio. Opción, 32(13), 228-254. Disponible en https://produccioncientificaluz.org/index.php/opcion/article/view/21598/21403

Chang, E. (2016b). Strategic Thought and TIC as Tools of Management of Neighborhood Grocery Stores' Owner. International Journal of Control Theory and Applications, 9(44), 175-181. Disponible en https://www.researchgate.net/publication/317168992_Strategic_Thought_and_TICas_Tools_of_Management_ of_Neighborhood_Grocery_Stores\%27_Owner

Chumaceiro, A. y Hernández, J. (2012). La legislación tributaria venezolana como promotora de la responsabilidad social empresarial. OIKOS, 16(33), 53-68. Recuperado de http:/ediciones.ucsh.cl/ojs/index.php/Oikos/article/ view/1041/982

De La Torre, M. (2018). Utilización del sector financiero para el lavado de dinero: perspectiva desde la legislación ecuatoriana. Jurídicas CUC, 14(1), 145-166. https://doi.org/10.17981/juridcuc.14.1.2018.07

Henríquez, G., Rada, J. \& Torrenegra, A. (2016). Medición de variables psicológicas, económicas y sociales para identificar factores ocultos de los emprendedores en Barranquilla. Económicas CUC, 37(1), 179-202. https://doi.org/10.17981/ econcuc.37.1.2016.08

Hernández, J., Meléndez, Y., Chumaceiro, A. y Gil, X. (2017). Cultura democrática, paz y convivencia social en Colombia. Abordaje desde una Escuela para Ciudadanos. En, J. Hernández, A. Ramírez y J. Barboza (Comp.), Conflictos y pos conflictos. Pasado y presente en América latina y el Caribe, caso Colombia (pp. 40-58). Cabimas: UNERMB. https://doi.org/10.21892/9789804270178

Hernández, J., Alvarado, L. \& Velazco, J. (2013). Participación ciudadana, tecnologías de información- comunicación y opinión pública. TEACS, 5(11), 25-34. Recuperado de https://dialnet.unirioja.es/descarga/articulo/4736149.pdf

Hernández, M. y Buitrago, R. (2017). Rol del sinésteta organizacional en el manejo de la comunicación asertiva. Económicas CUC, 38(2), 61-76. https://doi. org/10.17981/econcuc.38.2.2017.05 
Jaimes, H., Bravo, S., Cortina, A., Pacheco, C. y Quiñones, M. (2009). Planeación estratégica de largo plazo: una necesidad de corto plazo. Pensamiento \& Gestión, (26), 191-213. Recuperado de http://rcientificas.uninorte.edu.co/index.php/ pensamiento/article/viewFile/877/521

Morín, E. (2004). Introducción al pensamiento complejo. México, D. F.: Gedisa.

Paredes-Chacín, J. (2017). Financial planning before the organizational perspective in cement companies of the Zulia State. Económicas CUC, 38(1), 105-132. https://doi.org/10.17981/econcuc.38.1.05

Paz, A., Harris, J. y Franco, F. (2016). Responsabilidad social gestión compartida con el emprendedor social en empresas mixtas del sector petrolero. Económicas CUC, 37(2), 47-68. https://doi.org/10.17981/econcuc.37.2.2016.03

Reales, A. (2016). La democracia representativa en el marco de los derechos humanos en Colombia. Juridicas CUC, 12(1), 9-16. Recuperado a partir de https:// revistascientificas.cuc.edu.co/juridicascuc/article/view/1112

Ramírez, R., Rincón, Y., Chacón, H. y Alemán, L. (2018). Indicadores de gestión de la responsabilidad social en las pequeñas y medianas empresas. En, Y. Rincón, J. Restrepo y J. Vanegas, Gestión Organizacional y Desarrollo Responsable en las PYME. Una Mirada Glocal (Tomo IV, Cap. 16. pp. 424-443). Medellín: Sello Editorial PUBLICAR-T del Tecnológico de Antioquia.

Rojas, C. (2016). Labor flexibilization in Colombia. Juridicas CUC, 12(1), 17-29. Retrieved from https://revistascientificas.cuc.edu.co/juridicascuc/article/view/1113

Senge, P. (1994). La quinta disciplina. El arte y la práctica de la organización abierta al aprendizaje. Buenos Aires: Granica.

\section{BIODATA}

Alexandra Camila Vásquez Sarmiento es estudiante del octavo semestre de Administración de Empresas de la Universidad de la Costa (Barranquilla, Colombia). https://orcid.org/0000-0002-4076-3104 